\title{
Liver Dysfunction in Patients with Systemic Lupus Erythematosus
}

\author{
Atsushi Takahashi, Kazumichi Abe, Rie Saito, Haruyo Iwadate, Ken Okai, \\ Fumiko Katsushima, Kyoko Monoe, Yukiko Kanno, Hironobu Saito, Hiroko Kobayashi, \\ Hiroshi Watanabe and Hiromasa Ohira
}

\begin{abstract}
Objective We aimed to define the clinical features of liver dysfunction in patients with systemic lupus erythematosus (SLE).

Methods The frequency and causes of liver dysfunction were examined in 206 patients with SLE.

Results Liver dysfunction was evident in 123 (59.7\%) of the 206 patients. Liver dysfunction in patients with SLE can be drug-induced (30.9\%) or caused by SLE itself (28.5\%), fatty liver (17.9\%), autoimmune hepatitis $(\mathrm{AIH})(4.9 \%)$, primary biliary cirrhosis $(2.4 \%)$, cholangitis $(1.6 \%)$, alcohol $(1.6 \%)$ or viral hepatitis $(0.8 \%)$, and it tends to be mild except when caused by $\mathrm{AIH}$. Values for aminotransferase were significantly increased when AIH was the cause, whereas alkaline phosphatase (ALP) and $\gamma$-glutamyl transpeptidase $(\gamma$ GTP) were significantly increased when AIH or drugs were the cause. The liver was already dysfunctional at the time of SLE onset in 56 (45.5\%) of 123 patients with liver dysfunction. Neurological involvement was more common among patients with than without liver dysfunction, whereas SLE activity and prognosis did not significantly differ between the two groups.

Conclusion Liver dysfunction in the presence of SLE can be caused by many factors, but when extant at the time of SLE onset, either SLE itself or drugs can be the cause. Autoimmune hepatitis should be considered when liver dysfunction is relatively severe.
\end{abstract}

Key words: SLE, liver dysfunction, autoimmune hepatitis, anti-ribosomal P antibody

(Intern Med 52: 1461-1465, 2013)

(DOI: 10.2169/internalmedicine.52.9458)

\section{Introduction}

Systemic lupus erythematosus (SLE) affects several organs, including the liver (1). Not only SLE itself, but also other factors such as drug toxicity, fatty infiltration and overlapping autoimmune liver disease can cause liver dysfunction (2-7). Although SLE is systemic, specific organs tend to become affected and liver dysfunction is frequently associated with specific issues (2-7). For example, liver dysfunction in the presence of SLE is difficult to distinguish from autoimmune hepatitis (AIH) because the laboratory findings are similar. In addition, patients with SLE are often treated with corticosteroids and immunosuppressive drugs.
As a result, these patients are at risk of developing de novo hepatitis B. Liver dysfunction in patients with SLE is typically mild and temporary, so the causes are often overlooked. This study examines the clinical features of liver dysfunction in patients with SLE, and problems associated with differential diagnoses and identifying causes.

\section{Materials and Methods}

\section{Patients}

We enrolled 206 patients diagnosed with SLE or followed up for a mean of 13.1 years at Fukushima Medical University Hospital between 1973 and 2012. Table 1 shows the 
Table 1. Clinical Findings of 206 Patients with SLE

\begin{tabular}{lc}
\hline Characteristics & SLE $(\mathrm{n}=206)$ \\
\hline Sex (M/F) & $37 / 169$ \\
Age at SLE onset (y) & $33.5 \pm 17.2$ \\
Age at liver dysfunction onset (y) & $37.5 \pm 17.3$ \\
Follow-up (y) & $13.1 \pm 9.2$ \\
SLEDAI & $12.7 \pm 7.4$ \\
Exanthema & $104(50.5 \%)$ \\
Photosensitivity & $62(30.1 \%)$ \\
Oral ulcers & $37(17.9 \%)$ \\
Leukopenia & $44(21.4 \%)$ \\
Thrombopenia & $29(14.1 \%)$ \\
Arthritis & $100(48.5 \%)$ \\
Serositis & $41(19.9 \%)$ \\
Lupus nephritis & $89(43.2 \%)$ \\
Neurologic involvement & $23(11.2 \%)$ \\
Cardiopulmonary involvement & $11(5.3 \%)$ \\
\hline Liver dysfunction & $123(59.7 \%)$ \\
\hline SLEDAI SLE disease activity index &
\end{tabular}

characteristics of the patients. Systemic lupus erythematosus was diagnosed based on the criteria described by the American College of Rheumatology (8).

\section{Study protocols}

Liver dysfunction was defined as elevated serum levels of alanine aspartate aminotransferase (AST) (normal, <32 IU/ L), alanine aminotransferase (ALT) (normal, $<42$ IU/L) or alkaline phosphatase (ALP) (normal, <359 IU/L) as well as $\gamma$-glutamyl transpeptidase $(\gamma$-GTP) (normal, $<48 \mathrm{IU} / \mathrm{L}) . \mathrm{Pa}-$ tients were classified as having liver dysfunction when levels of at least two of these enzymes were outside the normal range. Patients were categorized according to clinical diagnosis. Drug-induced liver damage was indicated by Japanese diagnostic scores above 5 (9). Structural abnormalities and fatty liver were evaluated by ultrasonography and computed tomography, and viral markers, hepatitis B surface antigen and hepatitis $\mathrm{C}$ antibody, were screened. Fatty liver induced by corticosteroid therapy was classified as fatty liver. Diagnoses of AIH and primary biliary cirrhosis (PBC) were based on the criteria provided by Alvarez and Berg (10) and Sasaki et al. (11), respectively. Liver dysfunction without any obvious cause other than SLE, or that which improved in parallel with recovery from SLE, was classified as liver disease associated with SLE. The liver was histologically assessed to differentially diagnose AIH and SLE-associated hepatitis. Liver dysfunction with positive anti-ribosomal $\mathrm{P}$ antibody or temporarily elevated serum aminotransferase was classified as liver dysfunction associated with SLE without histological assessment. If liver dysfunction was caused by more than factor, the main cause was defined according to laboratory and clinical findings including the clinical course. Liver dysfunction that was not examined or otherwise remained obscure was classified as "unknown". The timing of liver dysfunction was compared by cause.
The initial diagnosis of SLE was defined as "SLE onset". White blood cell (WBC) counts, as well as values for CH50 and anti-double-stranded (ds) DNA antibodies were compared between patients with and without liver dysfunction at the onset of SLE. Some sera were assessed for reactivity against recombinant ribosomal P0 using ELISA kits (MBL Co., Ltd., Nagoya, Japan) according to the manufacturer's instructions. Liver samples from 25 patients with liver dysfunction were histologically evaluated. Clinical features and prognoses were compared between patients with and without liver dysfunction. Continuous variables are expressed as means \pm standard deviation (SD) and non-parametric values are shown as medians. Values of $\mathrm{p}<0.05$ were considered to be statistically significant.

\section{Results \\ Incidence of liver dysfunction in SLE and causes of liver dysfunction (Tables 1, 2)}

Liver dysfunction was evident in $123(59.7 \%)$ of 206 patients (28 (76\%) of 37 males and 95 (56\%) of 169 females) with SLE and the rate was significantly higher in males. The causes of liver dysfunction in patients with SLE were multifactorial and due to drug-induced liver damage $(30.9 \%)$, SLE itself $(28.5 \%)$, fatty liver (17.9\%), autoimmune hepatitis $(4.9 \%)$, PBC $(2.4 \%)$, cholangitis $(1.6 \%)$, alcohol $(1.6 \%)$ and viral hepatitis $(0.8 \%)$. The major causative agent of drug-induced liver dysfunction was antibiotics and the main causes of liver dysfunction in females and males were SLE $(30.2 \%)$ and drugs $(39.3 \%)$, respectively.

\section{Timing of liver dysfunction (Fig. 1)}

Liver dysfunction was extant at the onset of SLE in 56 $(45.5 \%)$ of 123 patients and developed largely at the onset of SLE in $28(80 \%)$ of 35. Fatty liver developed in 16 $(72.7 \%)$ of 22 patients after the onset of SLE. Drug-induced liver dysfunction developed essentially at the time of onset and during the course of SLE.

\section{Degree of liver dysfunction in SLE (Table 2)}

Liver dysfunction in patients with SLE was usually mild. However, the degree was determined by the cause. Levels of AST and ALT were highest in patients with AIH. Conversely, ALP and $\gamma$-GTP levels were highest in patients with AIH and drug-induced liver damage. Both the liver (AST or ALT) and the biliary ( $\gamma$-GTP or ALP) enzymes were elevated in $98(79.7 \%)$ of 123 patients. Either liver or biliary enzymes were elevated in $24(19.5 \%)$ and $1(0.8 \%)$ of 123 patients, respectively. The proportion of patients with only elevated liver enzymes was significantly lower in patients with liver dysfunction induced by drugs $(5.4 \%)$ than by SLE $(28.6 \%)$ or fatty liver $(36.4 \%)$. The patient with only elevated biliary enzymes had PBC. 
Table 2. Causes and Degrees of Liver Dysfunction

\begin{tabular}{|c|c|c|c|c|c|c|}
\hline Cause & $\mathrm{n}$ & $\%$ & AST (IU/L) & ALT (IU/L) & ALP (IU/L) & $\gamma$-GTP (IU/L) \\
\hline Drugs & 38 & 30.9 & $97.3 \pm 164.0$ & $150.3 \pm 275.5$ & $388.5 \pm 283.9^{\dagger}$ & $198.7 \pm 195.9^{\dagger}$ \\
\hline SLE & 35 & 28.5 & $90.5 \pm 97.3$ & $94.5 \pm 54.7$ & $239.4 \pm 17.2$ & $89.1 \pm 96.7$ \\
\hline Fatty liver & 22 & 17.9 & $40.0 \pm 14.8$ & $69.2 \pm 32.2$ & $193.0 \pm 96.7$ & $71.9 \pm 86.5$ \\
\hline $\mathrm{AIH}$ & 6 & 4.9 & $422.3 \pm 418.2^{*}$ & $485.0 \pm 405.4^{*}$ & $469.0 \pm 287.3^{\dagger}$ & $415.0 \pm 121.6^{\dagger}$ \\
\hline PBC & 3 & 2.4 & $41.3 \pm 18.7$ & $35.7 \pm 16.4$ & $420.7 \pm 139.2$ & $211.3 \pm 45.3$ \\
\hline Cholangitis & 2 & 1.6 & $86.5 \pm 84.1$ & $89.5 \pm 65.8$ & $691.0 \pm 544.5$ & $283.0 \pm 236.2$ \\
\hline Alcohol & 2 & 1.6 & $86.0 \pm 67.9$ & $54.5 \pm 53.0$ & $377.0 \pm 56.6$ & $115.0 \pm 79.2$ \\
\hline $\mathrm{HCV}$ & 1 & 0.8 & 40 & 42 & 286 & - \\
\hline Unknown & 14 & 11.4 & $33.4 \pm 16.9$ & $76.4 \pm 53.7$ & $199.3 \pm 35.6$ & $74.8 \pm 41.5$ \\
\hline Total & 123 & 100 & $97.9 \pm 170.1$ & $123.8 \pm 199.2$ & $302.3 \pm 225.3$ & $138.6 \pm 152.9$ \\
\hline
\end{tabular}

SLE: systemic lupus erythematosus, AIH: autoimmune hepatitis, PBC: primary biliary cirrhosis, HCV: hepatitis C virus, AST: alanine aspartate aminotransferase, ALT: alanine aminotransferase, ALP: alkaline phosphatase, $\gamma$-GTP: $\gamma$-glutamyl transpeptidase

${ }^{*} \mathrm{p}<0.05$ compared with drugs, SLE and fatty liver; ${ }^{\dagger} \mathrm{p}<0.05$ compared with SLE and fatty liver.

Same time as SLE onset

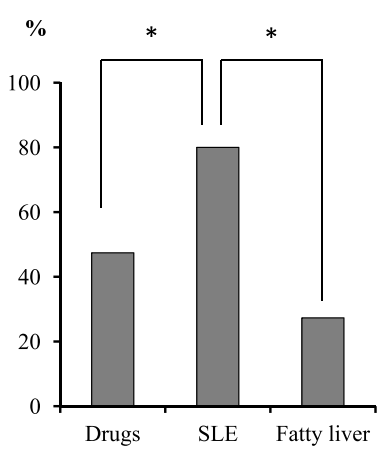

After SLE onset

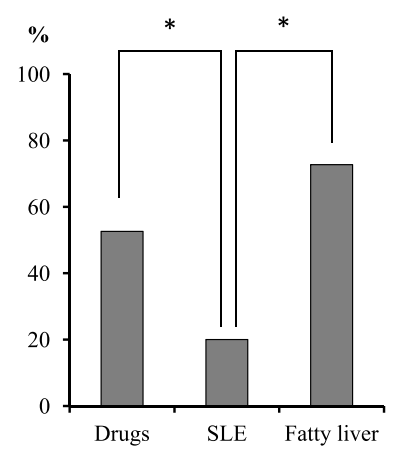

${ }^{*} \mathbf{p}<0.01$

Figure 1. Incidence of liver dysfunction.

\section{Relationship between liver dysfunction and SLE ac- tivity (Table 3)}

Numbers of WBC, levels of CH50, anti-dsDNA antibody titers at SLE onset and SLEDAI did not significantly differ between patients with and without liver dysfunction.

\section{Differential diagnosis of AIH-SLE overlap and SLE- induced liver dysfunction}

Levels of AST and ALT were significantly higher in patients with AIH-SLE overlap than in those with SLEinduced liver dysfunction (Table 2). We obtained liver biopsies from 25 patients and classified the causes of liver dysfunction as SLE (16), AIH (6) and PBC (3). Histological assessment of liver biopsies showed that liver dysfunction was caused by SLE in 14 (87.5\%) of 16 patients with nonspecific reactive hepatitis and in two with acute hepatitis. All patients with AIH-SLE overlap and $2(33.3 \%)$ of 6 with cirrhosis had interface hepatitis. Anti-ribosomal P antibody was positive in $11(64.7 \%)$ of 17 patients with liver dysfunction caused by SLE and in $2(33.3 \%)$ of 6 with AIHSLE overlap. Titers of anti-ribosomal $\mathrm{P}$ antibodies did not significantly correlate with levels of AST, ALT, ALP and $\gamma$ GTP.

Table 3. Relationships between Liver Dysfunction and SLE Activity

\begin{tabular}{lccc}
\hline & \multicolumn{2}{c}{ Liver dysfunction } & p value \\
& + & - & \\
\hline WBC $(\mu / \mathrm{mL})$ & $4,930 \pm 2,541$ & $4,621 \pm 2,109$ & 0.6793 \\
CH50 & $24.5 \pm 12.4$ & $20.3 \pm 9.3$ & 0.2852 \\
Anti-dsDNA antibody & $246.3 \pm 449.9$ & $322.1 \pm 619.5$ & 0.6496 \\
SLEDAI & $13.5 \pm 7.9$ & $11.7 \pm 6.7$ & 0.2105 \\
\hline
\end{tabular}

WBC: white blood cells, CH50: complement activity, Anti-dsDNA: antibody anti-double-stranded deoxyribonucleic acid antibody, SLEDAI: SLE disease activity index

\section{Incidence of liver dysfunction and other symptoms in patients with SLE (Fig. 2).}

We compared the clinical characteristics between SLE in patients with and without liver dysfunction. The incidence of nervous system involvement was significantly higher in patients with than without liver dysfunction. However, the incidence of exanthema, oral aphthae, photosensitivity, glomerulonephritis and hematological, musculoskeletal, or cardio-pulmonary involvement did not significantly differ between the two groups. The incidence of nervous system involvement was significantly higher in patients with liver dysfunction caused by SLE than in those without liver dysfunction (data not shown).

\section{Survival rates of SLE patients with or without liver dysfunction (Fig. 3)}

Survival rates did not significantly differ between the patients with and without liver dysfunction. Although a few patients had end-stage liver disease with portal hypertension and cirrhosis, none of the patients died due to liver failure. The main cause of death was infection or cancer.

\section{Discussion}

The reported incidence of liver dysfunction in patients with SLE ranges from $8.7 \%$ to $60 \%$ (2-7). We classified liver dysfunction based on a relatively lenient definition be- 


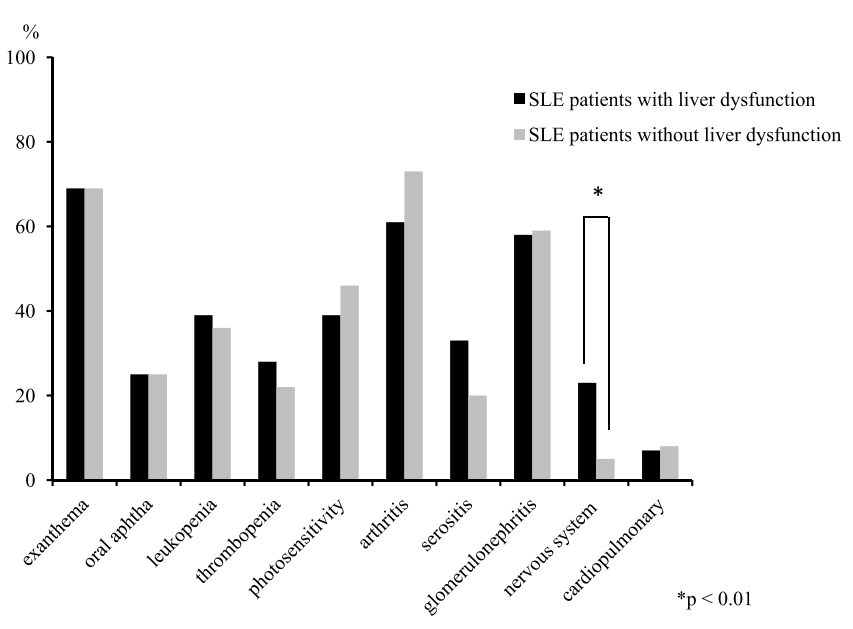

Figure 2. Incidence of liver dysfunction and other symptoms in SLE patients with and without liver dysfunction.

cause it is often masked by the immunosuppressive agents that are used to treat SLE. Therefore, we identified a higher incidence of liver dysfunction in patients with SLE than was previously thought. Although many factors can cause liver dysfunction, the timing of its onset or clinical course could provide useful information to help identify the cause. For example, fatty liver develops when SLE activity stabilizes under corticosteroid therapy. None of our patients developed liver dysfunction due to non-alcoholic steatohepatitis (NASH). However, the liver should be histologically assessed since corticosteroids can induce NASH. Liver dysfunction at the time of SLE onset is due to the SLE itself or drugs; thus, a history of drug administration must be determined.

The mechanisms of organ damage caused by SLE are complement deposition and vasculitis (12-14). In fact, previous reports have described vasculitis in liver specimens $(15,16)$. However, histological findings of the liver in patients with lupus hepatitis vary and the mechanisms of liver dysfunction remain unclear. Non-specific reactive hepatitis was the primary histological finding in this present study.

The degree of liver dysfunction was essentially mild, except when caused by AIH or viruses. De novo HBV has recently become a serious cause of liver dysfunction in patients undergoing chemotherapy or immunosuppressive therapy (17). Fulminant hepatic failure due to de novo HBV is lethal (18). Thus, patients with SLE should be tested for $\mathrm{HBV}$ to prevent fulminant liver failure, and HBV core as well as HBV surface antibodies must be assessed in patients who are HBV antigen-negative. Moreover, antiviral drugs should be administered according to the guidelines when HBV is confirmed (19).

Liver dysfunction caused by AIH in the present study was associated with relatively high levels of AST, ALT and ALP. Patients with AIH sometimes progress to fulminant hepatic failure (20) and thus a differential diagnosis between AIH and lupus hepatitis is important. Nonetheless, immunosup-

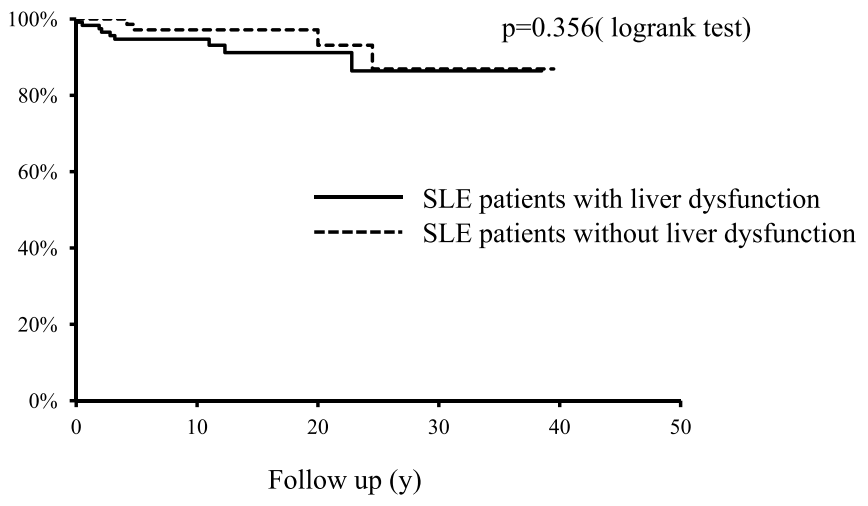

Figure 3. Survival rates of SLE patients with and without liver dysfunction.

pressive therapy makes more difficult the differential diagnosis. Anti-ribosomal $\mathrm{P}$ antibody is not only a marker of central nervous system (CNS) lupus, but also of lupus hepatitis (21-24). We found a higher positive rate of antiribosomal $\mathrm{P}$ antibody in patients with liver dysfunction caused by SLE. However, some patients with AIH-SLE overlap or AIH alone were also positive, and therefore a histological assessment of the liver is essential for differentially diagnosing $\mathrm{AIH}$ and SLE-associated hepatitis. We cannot deny the possibility without histological examination that SLE-associated hepatitis might have been AIH in the present study. If liver dysfunction persists, a liver biopsy should be assessed to differentially diagnose not only AIH and SLEassociated hepatitis but also other causes.

Many organs are affected by SLE and the features of liver dysfunction and other effects of SLE have not yet been clarified. The present study found a significantly higher incidence of nervous system involvement in patients with liver dysfunction, mostly caused by drugs $(22.9 \%)$ and SLE itself $(20.0 \%)$. Disorders of the nervous system, especially those of the CNS, are serious and complex and require treatment with several drugs. Mechanisms other than vasculitis might be associated with CNS lupus and lupus hepatitis because anti-ribosomal P antibody is a common marker of both (25). Moreover, the absence of a correlation between SLE activity and the development of liver dysfunction at SLE onset might support the above notion.

Almost all patients in the present study with SLE were treated with corticosteroids and their liver dysfunction improved. Patients with PBC were treated with ursodeoxycholic acid in addition to the corticosteroid. The causative drug was stopped when liver dysfunction was drug-induced. Patients with fatty liver were treated with diet and exercise. Survival rates did not significantly differ between patients with and without liver dysfunction. Therefore, although liver dysfunction in most patients with SLE does not require specific treatment, the cause of the dysfunction should be investigated to avoid overlooking factors such as AIH or de novo HBV that could lead to fatal liver failure.

In conclusion, liver dysfunction in patients with SLE is associated with specific tendencies that could indicate cause. 
The features of liver dysfunction and its causes need to be elucidated and considered before treating SLE.

The authors state that they have no Conflict of Interest (COI).

\section{References}

1. Cervera R, Khamashta MA, Font J, et al; The European working party of systemic lupus erythematosus. Systemic lupus erythematosus: clinical and immunologic patterns of disease expression in a cohort of 1,000 patients. The European Working Party on Systemic Lupus Erythematosus. Medicine (Baltimore) 72: 113-124, 1993.

2. Kojima H, Uemura M, Sakurai S, et al. Clinical features of liver disturbance in rheumatoid diseases: clinicopathological study with special reference to the cause of liver disturbance. J Gastroenterol 37: 617-625, 2002.

3. Miller MH, Urowitz MB, Gladman DD, Blendis LM. The liver in systemic lupus erythematosus. Q J Med 53: 401-409, 1984.

4. Chowdhary VR, Crowson CS, Poterucha JJ, Moder KG. Liver involvement in systemic lupus erythematosus: case review of 40 patients. J Rheumatol 35: 2159-2164, 2008.

5. Takahashi A, Abe K, Yokokawa J, et al. Clinical features of liver dysfunction in collagen disease. Hepatol Res 40: 1092-1097, 2010.

6. Runyon RA, LaBrecque DR, Asuras S. The spectrum of liver disease in SLE. Report of 33 histologically proved cases and review of the literature. Am J Med 69: 187-194, 1980.

7. Huang D, Aghdassi E, Su J, et al. Prevalence and risk factors for liver biochemical abnormalities in Canadian patients with systemic lupus erythematosus. J Rheumatol 39: 254-261, 2012.

8. Tan EM, Cohen AS, Fries JF, et al. The 1982 revised criteria for the classification of systemic lupus erythematosus. Arthritis Rheum 25: 1271-1277, 1982.

9. Takikawa H, Takamori Y, Kumagi T, et al. Assessment of 287 Japanese cases of drug induced liver injury by the diagnostic scale of the International Consensus Meeting. Hepatol Res 27: 192-195, 2003.

10. Alvarez F, Berg PA, Bianchi FB, et al. International Autoimmune Hepatitis Group Report: review of criteria for diagnosis of autoimmune hepatitis. J Hepatol 31: 929-938, 1999.

11. Sasaki H, Inoue K, Higuchi K, et al. Primary biliary cirrhosis in Japan: nation survey by the subcommittee on Autoimmune Hepatitis. Gastroenterol Jpn 20: 476-485, 1985.

12. Manderson AP, Botto M, Walport MJ. The role of complement in the development of systemic lupus erythematosus. Annu Rev Immunol 22: 431-456, 2004.
13. Calamia KT, Balabanova M. Vasculitis in systemic lupus erythematosis. Clin Dermatol 22: 148-156, 2004.

14. Sultan SM, Ioannou Y, Isenberg DA. A review of gastrointestinal manifestations of systemic lupus erythematosus. Rheumatology (Oxford) 38: 917-932, 1999.

15. Matsumoto T, Kobayashi S, Shimizu H, et al. The liver in collagen disease: pathologic study of 160 cases with particular reference to hepatic arteritis, primary biliary cirrhosis, autoimmune hepatitis and nodular regenerative hyperplasia of the liver. Liver 20: 366-373, 2000.

16. Matsumoto T, Yoshimine T, Shimouchi K, et al. The liver in systemic lupus erythematosus: pathologic analysis of 52 cases and review of Japanese Autopsy Registry Data. Hum Pathol 23: 11511158, 1992.

17. Hoofnagle JH. Reactivation of hepatitis B. Hepatology 49: s156s165, 2009.

18. Umemura T, Tanaka E, Kiyosawa K, Kumada H. Japan de novo Hepatitis B Research Group. Mortality secondary to fulminant hepatic failure in patients with prior resolution of hepatitis B virus infection in Japan. Clin Infect Dis 47: e52-e56, 2008.

19. Tsubouchi H, Kumada H, Kiyosawa K, Kumada H. Japan de novo Hepatitis B Research Group. Prevention of immunosuppressive therapy or chemotherapy-induced reactivation of hepatitis B virus infection-joint report of the intractable liver disease study group of japan and the Japanese study group of the standard antiviral therapy for viral hepatitis. Kanzo 50: 38-42, 2009 (in Japanese, Abstract in English).

20. Miyake Y, Iwasaki Y, Terada R, et al. Clinical characteristics of fulminant-type autoimmune hepatitis: an analysis of eleven cases. Aliment Pharmacol Ther 23: 1347-1353, 2006.

21. Arnett FC, Reichlin M. Lupus hepatitis: an under-recognized disease feature associated with autoantibodies to ribosomal P. Am J Med 99: 465-472, 1995.

22. Hulsey M, Goldstein R, Scully L, Surbeck W, Reichlin M. Antiribosomal $\mathrm{P}$ antibodies in systemic lupus erythematosus: a case control study correlating hepatic and renal disease. Clin Immunol Immunopathol 74: 252-256, 1995.

23. Yoshio T, Masuyama JI, Minota S, et al. A close temporal relationship of liver disease to antiribosomal P0 protein antibodies and central nervous system disease in patients with systemic lupus erythematosus. J Rheumatol 25: 681-688, 1998.

24. Ohira H, Takiguchi $J$, Rai $T$, et al. High frequency of antiribosomal $\mathrm{P}$ antibody in patients with systemic lupus erythematosus-associated hepatitis. Hepatol Res 28: 137-139, 2004.

25. Toubi E, Shoenfeld Y. Clinical and biological aspects of anti-Pribosomal protein autoantibodies. Autoimmun Rev 6: 119-125, 2007.

(C) 2013 The Japanese Society of Internal Medicine http://www.naika.or.jp/imonline/index.html 